\title{
Java in Biology
}

\section{Uttara Bidwaikar*}

Lecturer in Biomedical Engineering, India

*Corresponding author: Uttara Bidwaikar, India

Submission: 眥 October 17, 2017; Published: 眥 November 29, 2017

\section{Opinion}

Java is a user friendly language. Coding in biology using Java is easier as compared with other languages. Classes and Inheritance have made possible longer and multiple coding. Gene coding and matching using Java would be easier as compared to C. Object oriented programming makes objects instance of classes which can be invoked using objects. Also, web applications can be developed in different colors linking DBMS, Java and HTML.

Function calls at runtime than at compile time using pointers (virtual functions and abstract class derived from base class) saves modification of software. Connectivity of JDBC enables execution of distributed applications across multiple host systems using RMI that is implemented on middle tier of 3 tier architecture framework to invoke an object of a method present on other JVM.

Some examples are structured language/Program manager, top to down design abstract data structures, Grid layout for transfer of an image object from a remote server to client using RMI, Data structures like linked lists, objects stacks, queues, trees etc. Programming verification and texting efficieny and analysis of algorithm. Java is hence a better language with all features that enables coding, database management and server -client handling. 